\title{
Differential expression profiles of sense and antisense transcripts between HCV-associated hepatocellular carcinoma and corresponding non-cancerous liver tissue
}

\author{
KENTARO NAGAI ${ }^{1}$, KEISUKE KOHNO ${ }^{1}$, MITSURU CHIBA ${ }^{2}$, SUGIRU PAK $^{1}$, SOICHIRO MURATA $^{1}$, \\ KIYOSHI FUKUNAGA ${ }^{1}$, AKIHIKO KOBAYASHI ${ }^{1}$, HIROSHI YASUE ${ }^{3}$ and NOBUHIRO OHKOHCHI ${ }^{1}$ \\ ${ }^{1}$ Department of Surgery, Graduate School of Comprehensive Human Sciences, University of Tsukuba, \\ Tsukuba, Ibaraki 305-8575; ${ }^{2}$ Department of Biomedical Sciences, Division of Medical Life Sciences, \\ Hirosaki University Graduate School of Health Sciences, Hirosaki, Aomori 036-8564; ${ }^{3}$ Animal Genome \\ Research Unit, National Institute of Agrobiological Sciences, Tsukuba, Ibaraki 305-0901, Japan
}

Received December 5, 2011; Accepted January 20, 2012

DOI: 10.3892/ijo.2012.1382

\begin{abstract}
Recent studies have demonstrated that natural antisense transcripts, which are complementary sequences to messenger RNA, have important cellular functions such as the stabilization and silencing of mRNA. However, the possible contribution of antisense transcripts in hepatocellular carcinoma (HCC) development has not been described. Therefore, we simultaneously investigated the sense and antisense transcripts of HCC and non-cancerous tissues to explore the possible contribution of antisense transcripts to HCC progression. RNA was prepared from $15 \mathrm{HCV}$-associated HCCs and from 6 corresponding non-cancerous tissues and was subjected to expression profile analysis of sense and antisense transcripts using a human custom microarray. Differential expression of 161 sense and 25 antisense transcripts was observed with more than 2-fold between HCC and non-cancerous tissue ( $\mathrm{p}<0.001)$. The expression of the sense and antisense transcripts was used to cluster cancer and non-cancerous tissues, and the cancer and non-cancerous tissues were found to be clearly separated into different clusters. Additionally, the sense and antisense expression profiles were analyzed with regard to HCC differentiation $(\mathrm{p}<0.001)$, resulting in 71 sense and 43 antisense transcripts. These unique transcripts did not overlap with those found in the discrimination of HCC from non-cancerous tissues. When the HCC tissues were clustered by transcript expression, the antisense transcripts resulted in clustering of HCC that was consistent with grouping based on histology. These findings
\end{abstract}

Correspondence to: Dr Nobuhiro Ohkohchi, Department of Surgery, Graduate School of Comprehensive Human Sciences, University of Tsukuba, 1-1-1 Tennodai, Tsukuba, Ibaraki 305-8575, Japan

E-mail: nokochi3@md.tsukuba.ac.jp

Key words: natural antisense transcripts, human hepatocellular carcinoma, microarray, gene expression profile, tumor differentiation strongly indicate that the antisense transcripts together with the sense transcripts are involved in liver tumorigenesis.

\section{Introduction}

Hepatocellular carcinoma (HCC) is the fifth most common cancer in the world and the third cause of cancer-related deaths (1). However, only the $30 \%$ of patients who are at a very early stage of HCC receive benefits from curative therapies (2) and achieve 5-year survival rates of 50-70\% (3); the remaining 70\% of patients receive palliative treatments. Therefore, the detection of HCC at very early stages is a prerequisite for higher survival rates. In addition, the degree of differentiation of HCC is relevant to patient prognosis after curative treatments (4). In order to achieve early detection and a more accurate prognosis, $\mathrm{HCC}$ has been investigated in a variety of aspects such as histology, cytology, and biochemistry.

The advent of microarray analysis and mass-scale sequencing technologies has enabled the gene-expression profiling of cells, which has led us to compare the profiles of HCC with those of non-cancerous liver tissues. Previously, mRNA (5) and microRNA (6-8) have been reported to be biomarkers distinguishing HCC from non-cancerous liver tissues $(9,10)$. Despite such progress, the characterization of $\mathrm{HCC}$ and the prognosis of post-curative treatments using these biomarkers are still insufficient for practical use.

Recently, non-coding RNA such as microRNA, Piwiinteracting RNA, small nuclear RNA, and natural antisense transcripts have received attention due to their regulatory effects on genes (11-14). Antisense transcripts, a non-coding RNA species complementary to other intracellular RNA $(15,16)$, have been systematically identified in mammalian species (17); it has been indicated by widespread transcriptome analysis that up to $70 \%$ of human transcripts have antisense partners (18). Recent studies have reported that antisense transcripts are associated with a variety of gene regulation in eukaryotes $(19,20)$. For example, in Alzheimer's disease, BACE1 antisense transcripts form an RNA duplex with BACE1 mRNA and increase the stability of BACE1 mRNA (19). In leukemia, CDKN2B (also 
called p15) antisense transcripts may be a trigger for heterochromatin formation in tumor suppressor genes through the inverse relation between p15 sense and antisense expression (20).

However, a comprehensive antisense transcript analysis in human HCC samples has not yet been reported. In this study, we investigated the sense and antisense transcript profiles in HCC and non-cancerous liver tissues using a custom microarray in order to determine the possible involvement of these transcripts in liver tumorigenesis.

\section{Patients and methods}

Patients and samples. HCCs were resected from HCV-positive patients at the University of Tsukuba Hospital, frozen in liquid nitrogen, and stored at $-80^{\circ} \mathrm{C}$. The $\mathrm{HCC}$ specimens were collected from 15 patients who underwent surgical resection from August, 2006 through March, 2009. The study was approved by the hospital ethics committee and informed consent was obtained from all patients. Prior to RNA preparation, tissues were subjected to histological examination to select HCCs and non-cancerous sections. As listed in Table I, the sections were obtained from 15 HCCs and 6 non-cancerous tissues as samples for microarray analysis.

Total RNA extraction. Total RNA was isolated from frozen samples using Isogen reagent (Nippon Gene, Tokyo, Japan) according to the manufacturer's instructions. RNA concentrations were determined by measuring the absorbance at 260/280 nm on the NanoDrop Spectrophotometer (NanoDrop Technologies, Wilmington, DE) according to the manufacturer's instructions. The integrity of the total RNA was examined using an Agilent 2100 Bioanalyzer (Agilent Technologies, Santa Clara, CA) and an RNA 6000 Nano LabChip kit (Agilent Technologies).

Probe design of the custom microarray. One of the steps in microarray gene expression analysis is to use in situ hybridization to investigate gene expression sites in tissues. The probe size for in situ hybridization should be approximately 120 nucleotides (nt) to obtain a satisfactory hybridization signal/noise ratio. Since the copy numbers of gene transcripts have been shown to vary depending on the gene region (21), probe sequences for microarray and for in situ hybridization should be selected in the same gene region in order to interpret the results of microarray in combination with in situ hybridization. Therefore, $120 \mathrm{nt}$ sequences were first selected from human ORF sequences (Build35) for use as a probe for in situ hybridization (Genetyx, Tokyo, Japan). The selected sequences were confirmed to be unique in the human genomic sequence by BLAST analysis, and were then submitted to the Agilent server (Agilent Technologies) to design $60 \mathrm{nt}$ sequences for use as microarray probes. The sense and antisense sequences of $60 \mathrm{nt}$ were arranged in an Agilent 44 K x 4 system (20882 ORFs: Agilent eArray Design ID 19052 produced by Tsukuba Gene Technology Laboratories, Tsukuba, Japan) (Agilent Technologies).

Microarray analysis. Cyanine 3 (Cy3)-labeled cDNA was synthesized from $10 \mu \mathrm{g}$ total RNA of HCC and non-cancerous samples using a LabelStar Array kit (Qiagen, Valencia, CA),
Cy3-dUTP (GE Healthcare, Fairfield, CT), and a random nonamer primer. The labeled cDNA was hybridized with probe sequences on an Agilent $44 \mathrm{~K}$ x 4 human sense and antisense custom microarray slide (22) in a hybridization solution prepared with In Situ Hybridization kit Plus (Agilent Technologies), following the manufacturer's instructions. The $\mathrm{Cy} 3$ fluorescence signal images on the slides were obtained by a DNA microarray scanner (Agilent Technologies) and processed using the Feature Extraction version 8.1 software based on instruction from Agilent Technologies. The expression data thus obtained were processed using Gene Spring GX version 11.5 software (Agilent Technologies) to perform a log transformation and normalization to the 75 percentile of all values on the respective microarrays, followed by normalization of the median expression level of all samples. Additionally, the normalized gene expression data were filtered on flags, and only those genes classified as either flag-Present or flag-Marginal in $>70 \%$ of all samples, were allowed to pass the filter.

The expression profiles of the samples were compared using unpaired t-tests (with Benjamini-Hochberg FDR correction for unequal variances) as described in the Results section. Two-dimensional hierarchical clustering based on Euclidean distance measures was performed using Ward's method. Results are visualized with the help of heat maps and dendrograms. The heat maps show color-coded expression levels; the color gradation from red to blue indicates the expression levels from high to low. Sample trees are drawn horizontally and gene trees were drawn vertically. To identify genes with statistically significant differences in tumors with varying degrees of differentiation, a one-way ANOVA with a p-value cutoff of 0.001 was performed. To characterize the trends of multigene expression, we used principal component analysis (PCA).

\section{Results}

Identification of sense and antisense transcripts differentially expressed between HCV-associated HCC and non-cancerous liver tissue. In order to determine the sense and antisense transcripts that are differentially expressed between HCC and non-cancerous liver tissue, total RNA was prepared as described in Patients and methods. To reduce background differences, samples were obtained only from HCV-positive patients. All total RNAs were shown to have 260/280 nm absorbance ratios of 1.8-2.0 and integrity values (RIN) ranging from 7.9-9.9. Based on the instruction for microarray analysis (Agilent Technologies), the quality of the RNA was determined to be appropriate for microarray analysis.

The total RNA from $15 \mathrm{HCC}$ tissues and 6 corresponding non-cancerous liver tissues (Table I) was subjected to microarray analysis as described in Patients and methods. It was determined that 186 transcripts were differentially expressed between HCC and non-cancerous liver tissues with a magnitude of more than 2-fold, based on an unpaired t-test ( $\mathrm{p}<0.001)$; 161 transcripts were sense sequences and the remaining 25 transcripts were antisense sequences (Table II). Of the 161 sense transcripts, 138 were found to be up-regulated, and the remaining 23 were down-regulated. Furthermore, of the 25 antisense transcripts, 18 were found to be up-regulated, and the remaining 7 were down-regulated. Upon examination for the presence of common genes, the sense transcript (mRNA) and antisense transcript of 
Table I. Characteristics of patients, as used for microarray analysis.

\begin{tabular}{lcccrrrrc}
\hline Sample no. & Age & Gender & Histology & AFP & PIVKA-II & $\begin{array}{c}\text { Fibrosis } \\
\text { score(F) }\end{array}$ & $\begin{array}{c}\text { Activity } \\
\text { score(A) }\end{array}$ & $\begin{array}{c}\text { Normal } \\
\text { tissue }\end{array}$ \\
\hline 35 & 79 & M & Mod & 71 & 2291 & 3 & 2 & - \\
36 & 74 & M & Mod & 10 & 23 & 3 & 2 & Yes \\
50 & 75 & M & Well & 8 & 33 & 4 & $1-2$ & - \\
56 & 70 & M & Well & 33 & 14 & 3 & 2 & Yes \\
59 & 75 & F & Mod & 263 & 37 & 3 & 2 & - \\
62 & 71 & M & Well & 39 & 184 & 3 & 2 & - \\
64 & 75 & M & Well & 41 & 11 & 4 & 2 & Yes \\
66 & 49 & M & Well & 11 & 97 & 4 & 2 & - \\
69 & 60 & M & Poor & 2 & 35 & 2 & 1 & - \\
73 & 69 & F & Mod & 762 & 68 & 4 & 2 & Yes \\
75 & 75 & M & Mod & 6 & 253 & 2 & 2 & - \\
77 & 54 & M & Poor & 5417 & 933 & - & - & - \\
79 & 57 & M & Poor & 258 & 149 & 4 & 2 & Yes \\
96 & 52 & M & Mod & 91 & 95 & 3 & 1 & Yes \\
98 & 71 & M & Mod & 46 & 32649 & 2 & 1 & - \\
\hline
\end{tabular}

F, fibrosis; A, activity; Mod, moderate.

A

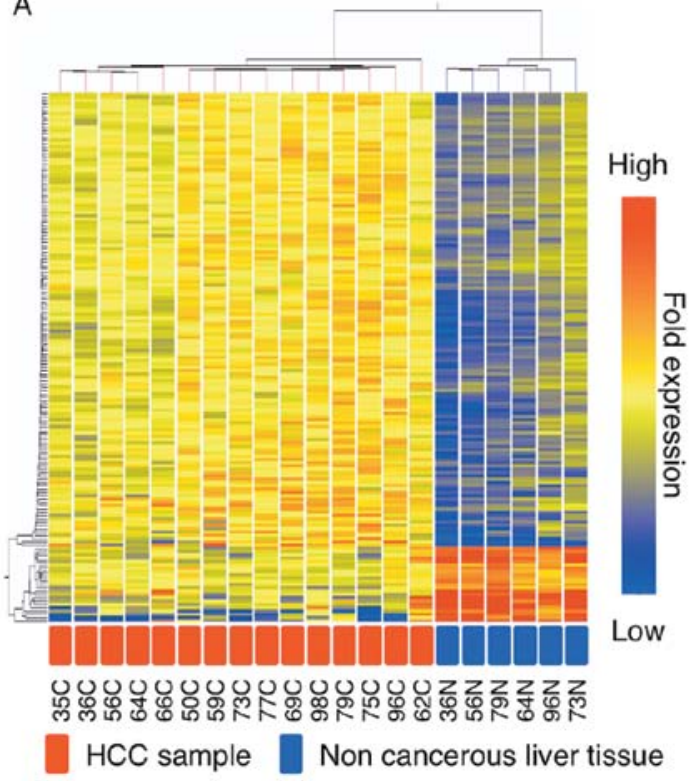

B

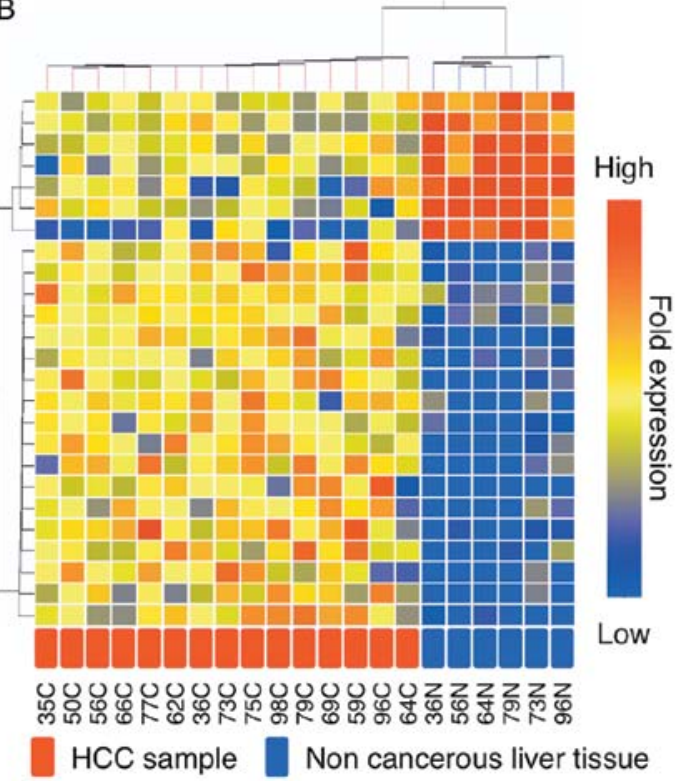

Figure 1. (A) A hierarchical cluster analysis using 161 up- and down-regulated sense transcripts of HCC and non-cancerous liver tissue samples. The heat maps show color-coded expression levels (the color gradation from red to blue indicates the expression levels from high to low). A blue box at the bottom of the image indicates non-cancerous samples; a red box indicates HCC samples. (B) A hierarchical cluster analysis using 25 up- and down-regulated antisense transcripts of HCC and non-cancerous liver tissue samples.

metadherin (MTDH) were both found to be up-regulated in HCCs.

Some of the 161 sense transcripts have been already demonstrated to be candidates for markers of HCC (23-30). This fact provided support for the assumption that the microarray results accurately reflect gene expression of HCC and non-cancerous tissues. In addition, analysis of the custom microarray for colorectal cancer produced results that were consistent with those of quantitative RT-PCR (22). Therefore, without verification of the array results by quantitative RT-PCR, we proceeded to the clustering analysis of the 161 sense and 25 antisense transcripts. HCC samples and non-cancerous tissue samples were separately clustered for sense transcripts as well as antisense transcripts as shown in Fig. 1A and B. These results demonstrated that expression profiles of sense and antisense transcripts varied between HCC and non-cancerous tissues. 
Table II. Twenty-five natural antisense transcripts differentially regulated between HCC and non-cancerous liver tissue.

\begin{tabular}{|c|c|c|c|c|}
\hline Accession no. & Gene symbol & Gene name & Fold change & Regulation \\
\hline NM001018136.1 & NME1-NME2 & NM23-LV & 4.0762205 & Up \\
\hline NM005896.2 & IDH1 & Isocitrate dehydrogenase $1\left(\mathrm{NADP}^{+}\right)$, soluble & 3.9515014 & Up \\
\hline NM000989.2 & RPL30 & Ribosomal protein L30 & 3.9422822 & Up \\
\hline NM016032.2 & ZDHHC9 & Zinc finger, DHHC-type containing 9 & 3.8781722 & Up \\
\hline NM024671.2 & ZNF768 & Zinc finger protein 768 & 3.800295 & Up \\
\hline NM198880.1 & QRICH1 & Glutamine-rich 1 & 3.7468915 & Up \\
\hline NM016645.2 & NGRN & Neugrin, neurite outgrowth associated & 3.6181226 & Up \\
\hline NM006808.2 & SEC61B & Sec61 $\beta$ subunit & 3.568331 & Up \\
\hline NM015289.2 & VPS39 & Vacuolar protein sorting 39 (yeast) & 3.4819472 & Up \\
\hline NM005005.1 & NDUFB9 & $\begin{array}{l}\text { NADH dehydrogenase (ubiquinone), } 9,22 \mathrm{kDa} \\
1 \beta \text { subcomplex }\end{array}$ & 3.4809504 & Up \\
\hline NM203298.1 & CHCHD1 & Coiled-coil-helix-coiled-coil-helix domain containing 1 & 3.3855672 & Up \\
\hline NM006070.4 & TFG & TRK-fused gene & 3.2429345 & Up \\
\hline NM181528.2 & NAT5 & $\mathrm{N}$-acetyltransferase 5 & 3.0416958 & Up \\
\hline NM001007027.2 & ALG8 & $\begin{array}{l}\text { Asparagine-linked glycosylation } 8 \text { homolog } \\
\text { (S. cerevisiae, } \alpha-1,3 \text {-glucosyltransferase) }\end{array}$ & 2.9185905 & Up \\
\hline NM006694.2 & JTB & Jumping translocation breakpoint & 2.889171 & Up \\
\hline XM001133555.1 & LOC729130 & $\begin{array}{l}\text { Similar to phosphodiesterase 4D interacting } \\
\text { protein isoform } 1\end{array}$ & 2.5856044 & Up \\
\hline NM001039712.1 & DEDD & Death effector domain containing & 2.037344 & Up \\
\hline NM178812.2 & MTDH & Metadherin & 2.027995 & Up \\
\hline NM012174.1 & FBXW8 & F-box and WD-40 domain protein 8 & 5.336158 & Down \\
\hline NM133507.2 & DCN & Decorin & 3.7978623 & Down \\
\hline NM000598.4 & IGFBP3 & Insulin-like growth factor binding protein 3 & 3.5457635 & Down \\
\hline NM001781.1 & CD69 & CD69 molecule & 3.4615583 & Down \\
\hline NM171825.1 & CAMK2A & $\begin{array}{l}\text { Calcium/calmodulin-dependent protein } \\
\text { kinase (CaM kinase) II } \alpha\end{array}$ & 2.8482397 & Down \\
\hline XR018502.1 & LOC644202 & Similar to $60 \mathrm{~S}$ ribosomal protein L12 & 2.426251 & Down \\
\hline NM015658.1 & NOC2L & Nucleolar complex associated 2 homolog (S. cerevisiae) & 2.349828 & Down \\
\hline
\end{tabular}

The sense and the antisense transcripts thus identified were further investigated using PCA analysis. As shown in Fig. 2A and $\mathrm{B}, \mathrm{HCC}$ and non-cancerous tissues were found to be well separated with the first principal component (PC1); contribution rates were 86.4 and $79.1 \%$ for sense and antisense transcripts, respectively. The contribution rates of the second principal component (PC2) were calculated to be 3.0 and $4.2 \%$ for sense and antisense transcripts, respectively. These findings together indicate that the PC1 was sufficient for the separate clustering of HCC and non-cancerous tissues.

When the constitution of PC1 and PC2 for sense transcripts was examined, respective transcripts were found to contribute to PC1 at nearly equal levels and to PC2 at various levels (data not shown). Similarly, with regards to the antisense transcripts, respective transcripts were found to contribute to $\mathrm{PC} 1$ at nearly equal levels and to PC2 at various levels (data not shown).

Identification of sense and antisense transcripts differentially expressed with respect to differentiation stages of HCCs. The histological differentiation levels of HCC have been demonstrated to be strongly related to prognosis of HCC (4). Therefore, we then examined whether the expression profiles distinguished the histological differentiation of HCC. The results of the microarray analysis were subjected to statistical analysis in terms of histological differentiation of HCCs (5 were well differentiated, 7 were moderately differentiated, and 3 were poorly differentiated) using the one-way ANOVA, followed by the Tukey HSD post-hoc test $(\mathrm{P}<0.001)$. The analysis allowed us to select 71 sense and 43 antisense transcripts. Of these transcripts, only one gene, a corticotropin releasing hormone binding protein (CRHBP), was present in both sense and antisense transcripts.

First, we performed clustering of the 15 HCCs using the 71 sense transcripts. As a result of the clustering shown in Fig. 3A, HCCs with the same differentiation stage except 36C HCC were clustered into the same group. The $36 \mathrm{C} \mathrm{HCC}$, which was shown to be moderately differentiated, was clustered with the well differentiated HCCs. The expression profiles of the 71 sense transcripts in the 15 HCCs were further investigated with PCA analysis, revealing that the HCCs were separated into groups depending on differentiation stages when PC1 (contribution 
A

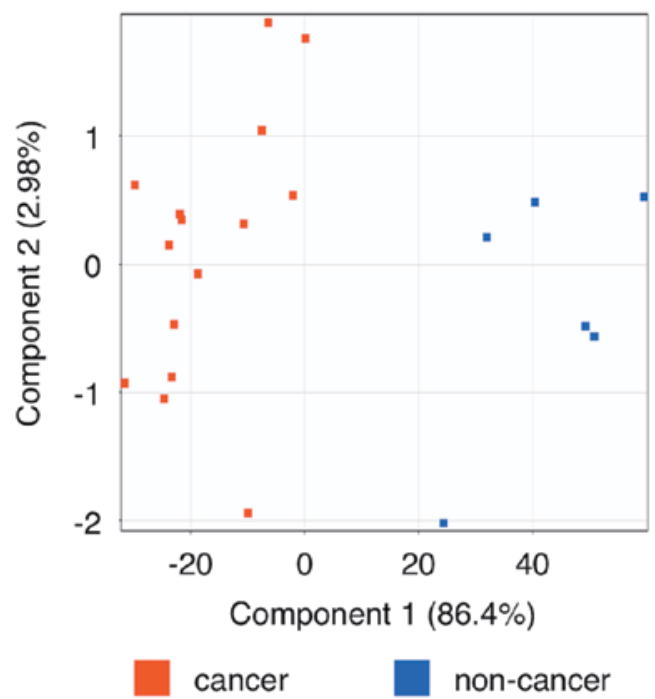

B

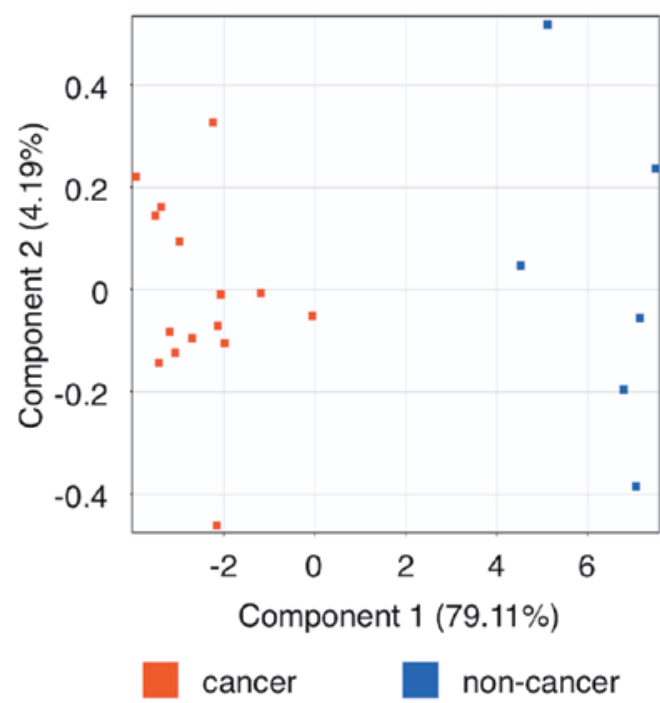

Figure 2. (A) A graphical presentation of principal component analysis (PCA) for 15 HCC samples and 6 corresponding non-cancerous samples using 161 sense transcripts based on PC1 (contribution ratio: 86.4\%) and PC2 (contribution ratio: 3.0\%). A blue box indicates non-cancerous samples; a red box indicates HCC samples. (B) A graphical presentation of principal component analysis (PCA) for 15 HCC samples and 6 corresponding non-cancerous samples using 25 antisense transcripts based on PC1 (contribution ratio: $79.1 \%$ ) and PC2 (contribution ratio: $4.2 \%$ ). A blue box indicates non-cancerous samples; a red box indicates HCC samples.

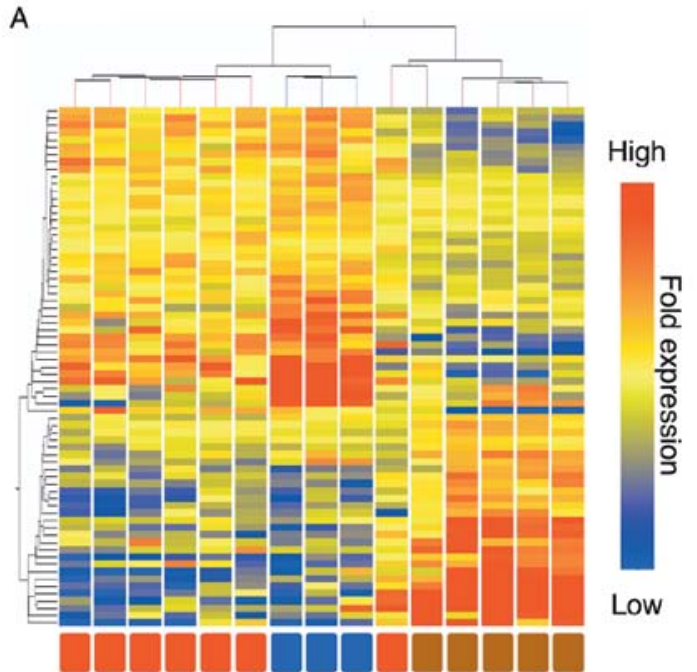

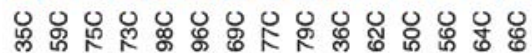
moderate poor

B

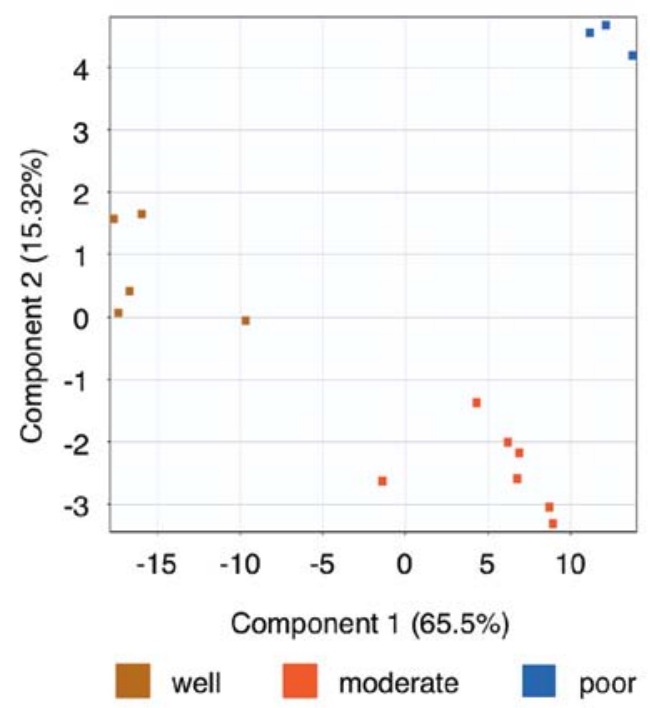

Figure 3. (A) A hierarchical cluster analysis using 71 sense transcripts of HCC and non-cancerous liver tissue samples. The heat maps show color-coded expression levels (the color gradation from red to blue indicates the expression levels from high to low). A red box at the bottom of the image indicates moderate differentiation; a blue box indicates poor differentiation, and a brown box indicates well differentiated sample. (B) A graphical presentation of principal component analysis (PCA) for 15 HCC samples using 71 sense transcripts based on PC1 (contribution ratio: $65.5 \%$ ) and PC2 (contribution ratio: 15.3\%). A brown box indicates well differentiated sample; a red box indicates moderate differentiation, and a blue box indicates poor differentiation.

rate: $65.5 \%$ ) and $\mathrm{PC} 2$ (contribution rate: $15.3 \%$ ) were used for scatter plot (Fig. 3B). For PC1, 58 transcripts were found to contribute to the component at nearly equal levels; the remaining 13 contributed to the component less than the 58 transcripts (data not shown). For PC2, contribution rates of the 71 transcripts were variable (data not shown).

Next, we performed clustering of the HCCs using the 43 antisense transcripts, revealing that HCCs with the same differentiation stages were clustered into the same group (Fig. 4A). As in the sense transcripts, the expression profiles of the 43 antisense transcripts were investigated with PCA analysis, which revealed the following: the HCCs were separated into groups depending on differentiation stage, when $\mathrm{PC} 1$ (contribution rate: $60.3 \%$ ) and $\mathrm{PC} 2$ (contribution rate: $17.6 \%$ ) were used for a scatter plot (Fig. 4B). For PC1, 32 transcripts were found to contribute to the component at nearly equal levels, 


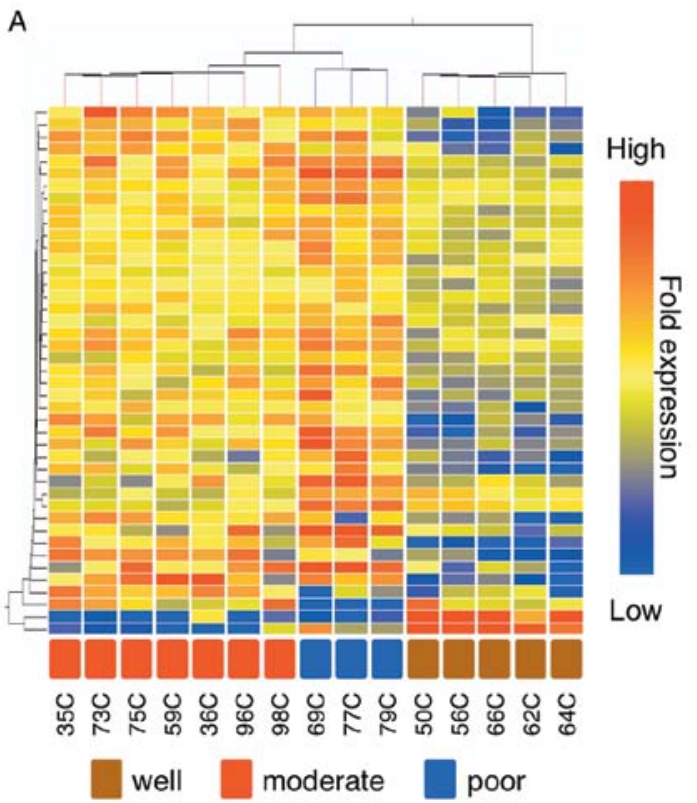

B

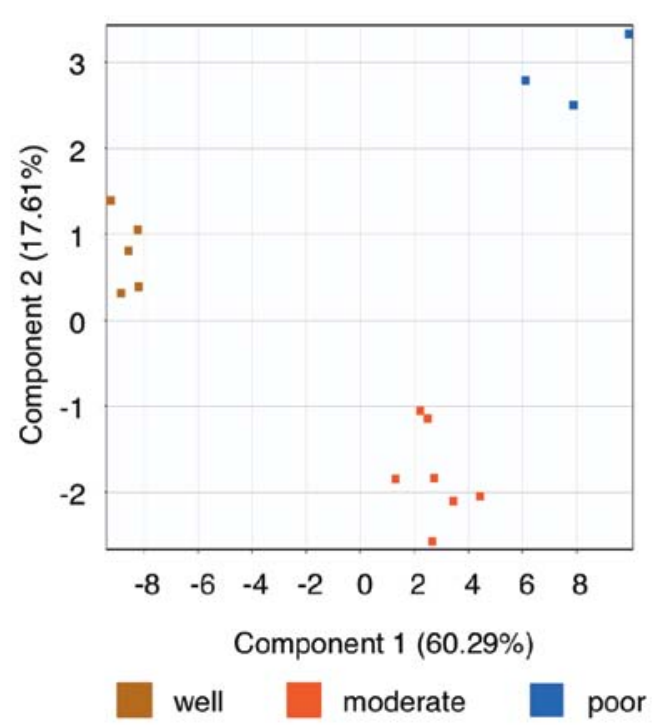

Figure 4. (A) A hierarchical cluster analysis using selected 43 antisense transcripts of $\mathrm{HCC}$ and non-cancerous liver tissue samples. The heat maps show colorcoded expression levels (the color gradation from red to blue indicates the expression levels from high to low). A red box at the bottom of the image indicates moderate differentiation; a blue box indicates poor differentiation, and a brown box indicates a well differentiated sample. (B) A graphical presentation of principal component analysis (PCA) for $15 \mathrm{HCC}$ samples using 43 antisense transcripts based on PC1 (contribution ratio: 60.3\%) and PC2 (contribution ratio: 17.6\%). A brown box indicates a well differentiated sample; a red box indicates moderate differentiation, and a blue box indicates poor differentiation.

and the remaining 11 contributed to the component less than the 32 transcripts (data not shown). For PC2, contribution rates of the 43 transcripts were variable (data not shown).

The findings obtained in the present study are summarized as follows: The HCCs were clearly distinguished from noncancerous liver tissues by clustering and PCA analyses using the expression profiles of the 161 sense and the 25 antisense transcripts. Of the transcripts, only one gene, MTDH, was altered in the sense as well as antisense transcripts. In addition, the grouping of HCCs based on their differentiation stages determined by histological examination was consistent with that based on the clustering and PCA analyses using the expression profiles of the 43 antisense transcripts, and essentially with that using the 71 sense transcripts. In these transcripts, only one gene, CRHBP, was changed in the sense as well as antisense transcripts. When the transcripts selected for discrimination of cancer from normal tissues were compared with those for HCCs differentiation, no transcripts were found to be overlapping.

\section{Discussion}

Many studies in the past decade have shown that epigenetic changes can play an important role in hepatocarcinogenesis (31-33). Several studies have focused on the expression profiling of HCC using microarrays, and discovered sense transcripts $(5,28,34-37)$ and miRNAs (6-10) distinguish cancerous from non-cancerous tissues. These studies revealed that there are different expression patterns between cancerous and noncancerous tissues. On the other hand, some studies have reported that there are NATs expression change in Alzheimer's disease (19), leukemia (20), CRC (22), breast cancer (38). In the present study, we simultaneously investigated the profiles of sense and antisense transcripts of HCC and non-cancerous liver tissues to explore the possible contribution of antisense transcripts to HCC progression. To our knowledge, this is the first study showing the comprehensive analysis of NATs in human HCC and non-cancerous tissues in order to seek specific biomarkers for HCC and to clarify the mechanism of HCC development.

Gene expression profiles of HCC on mRNA and miRNA have been reported with significantly different expression levels between cancerous and non-cancerous tissues $(5,28,34-37)$. We investigated the antisense transcripts that distinguish cancerous tissues from non-cancerous tissues in $\mathrm{HCV}$-associated liver. Our result indicated that it is possible to detect cancer-specific expression patterns using antisense transcripts. In this study, we also investigated the profile of sense transcripts, and expression changes in the gene, DPT (39), CXCL14 (40), HAMP (41), MAGE1 A (42) were consistent with results of previous studies. In contrast to genes discussed above, some genes that were reported previously were not recognized in the present study. This discrepancy may be due to different sample size and different infected virus types of the patients.

Although the function of antisense transcripts is not yet well understood, recent studies have shown that antisense transcripts play a role to stabilize mRNA (43), and/or silencing mRNA (20). Moreover, Faghihi et al reported antisense transcript-mediated inhibition of miRNA function (44). In the present study, we investigated the expression levels of sense-antisense pairs in cancerous and non-cancerous tissue. We observed both positive and negative correlations of sense-antisense expression levels ( $\mathrm{p}<0.05$, fold change $>2.0$ ), and $>75 \%$ (data not shown) were positive correlation. This result was consistent with the results of Grigoriadis et al (38) reported in breast tumors and suggests that antisense transcripts both stabilize and silence of mRNA function.

Multistep tumorigenesis was reported in colorectal cancer (45). HCC is also characterized by multistep process of 
tumor progression by histopathological analyses $(46,47)$. Nakanishi et al reported that tumor differentiation is one of the risk factors for poor prognosis (4). Midorikawa et al reported several genes are likely to be associated with dedifferentiation of HCC (48). We analyzed gene expression profiles and found differentially expressed antisense transcripts with strong relation to the histological differentiations. In clustering analysis of this study, samples were separately clustered into two groups i.e., well differentiated cluster, and moderately plus poorly differentiated cluster. Patient no. 36 , whose cancerous tissue was histologically classified as moderately differentiated HCC, was clustered with the well differentiated HCCs by sense transcripts (Fig. 3A), but this patient was clustered with moderately differentiated HCCs according to antisense transcripts (Fig. 4A). We re-inspected the histological findings of the patient, but diagnosis of moderately differentiated carcinoma was confirmed. These findings combined with the histological examination indicated that the antisense transcripts are a more suitable parameter for the determination of HCC profile than the sense transcripts. However, since there is still a possibility that, although the patient was grouped into the moderately differentiated cluster, the character of the patient might be different from the other moderately differentiated HCCs, the patient should be carefully followed after treatment. This result described above indicates that analysis of HCC by antisense transcripts would stratify patients more accurate than by sense transcripts.

In the present study, not only sense transcripts but also antisense transcripts were demonstrated to be involved in liver tumorigenesis and de-differentiation of HCC cells. These results indicate that expression profile of antisense transcripts contribute to prediction of prognosis and recurrence types, and to decisions for treatment strategy such as surgery, chemotherapy, and transcatheter arterial embolization. As for the selection of biomarkers for clinical use, a large number of HCC samples should be examined to select reliable biomarkers and analyze the relationship with prognosis and recurrent types in the future. In addition, analysis of mRNA in peripheral blood has been reported for biomarker discovery (49), therefore it is necessary to analyze expression profile of antisense transcripts in peripheral blood to find useful biomarkers.

Antisense transcripts have been demonstrated to be involved in gene regulation, but the function and mechanisms of antisense transcripts are still unclear. Functional analyses are required to define the mechanisms of tumorigenesis.

\section{Acknowledgements}

This study is supported in part by grants from the Ministry of Education, Culture, Sports, Science and Technology of Japan (MEXT). We thank Hideo Tanaka, Tomy Digital Biology Co., Ltd, Tokyo, for excellent support in GeneSpring GX software analysis.

\section{References}

1. El-Serag HB and Rudolph KL: Hepatocellular carcinoma: epidemiology and molecular carcinogenesis. Gastroenterology 132: 2557-2576, 2007.
2. Bruix J, Sherman M, Llovet JM, et al: Clinical management of hepatocellular carcinoma. Conclusions of the Barcelona-2000 EASL conference. European Association for the Study of the Liver. J Hepatol 35: 421-430, 2001.

3. Bruix J and Llovet JM: Major achievements in hepatocellular carcinoma. Lancet 373: 614-616, 2009.

4. Nakanishi K, Sakamoto M, Yamasaki S, Todo S and Hirohashi S: Akt phosphorylation is a risk factor for early disease recurrence and poor prognosis in hepatocellular carcinoma. Cancer 103: 307-312, 2005.

5. Okabe H, Satoh S, Kato T, et al: Genome-wide analysis of gene expression in human hepatocellular carcinomas using cDNA microarray: identification of genes involved in viral carcinogenesis and tumor progression. Cancer Res 61: 2129-2137, 2001.

6. Huang $\mathrm{XH}$, Wang Q, Chen JS, et al: Bead-based microarray analysis of microRNA expression in hepatocellular carcinoma: miR-338 is downregulated. Hepatol Res 39: 786-794, 2009.

7. Huang YS, Dai Y, Yu XF, et al: Microarray analysis of microRNA expression in hepatocellular carcinoma and non-tumorous tissues without viral hepatitis. J Gastroenterol Hepatol 23: 87-94, 2008.

8. Murakami Y, Yasuda T, Saigo K, et al: Comprehensive analysis of microRNA expression patterns in hepatocellular carcinoma and non-tumorous tissues. Oncogene 25: 2537-2545, 2006.

9. Ito H, Funahashi S, Yamauchi N, et al: Identification of ROBO1 as a novel hepatocellular carcinoma antigen and a potential therapeutic and diagnostic target. Clin Cancer Res 12: 3257-3264, 2006.

10. Midorikawa Y, Ishikawa S, Iwanari H, et al: Glypican-3, overexpressed in hepatocellular carcinoma, modulates FGF2 and BMP-7 signaling. Int J Cancer 103: 455-465, 2003.

11. Willingham AT and Gingeras TR: TUF love for 'junk' DNA. Cell 125: 1215-1220, 2006.

12. Knee R and Murphy PR: Regulation of gene expression by natural antisense RNA transcripts. Neurochem Int 31: 379-392, 1997.

13. Novina CD and Sharp PA: The RNAi revolution. Nature 430: 161-164, 2004.

14. Hannon GJ: RNA interference. Nature 418: 244-251, 2002.

15. Vanhee-Brossollet $C$ and Vaquero C: Do natural antisense transcripts make sense in eukaryotes? Gene 211: 1-9, 1998.

16. Lavorgna G, Dahary D, Lehner B, Sorek R, Sanderson CM and Casari G: In search of antisense. Trends Biochem Sci 29: 88-94, 2004.

17. Rosok O and Sioud M: Systematic identification of senseantisense transcripts in mammalian cells. Nat Biotechnol 22: 104-108, 2004.

18. Katayama S, Tomaru Y, Kasukawa T, et al: Antisense transcription in the mammalian transcriptome. Science 309: 1564-1566, 2005.

19. Faghihi MA, Modarresi F, Khalil AM, et al: Expression of a noncoding RNA is elevated in Alzheimer's disease and drives rapid feed-forward regulation of beta-secretase. Nat Med 14: 723-730, 2008

20. Yu W, Gius D, Onyango P, et al: Epigenetic silencing of tumour suppressor gene p15 by its antisense RNA. Nature 451: 202-206, 2008.

21. Clark TA, Schweitzer AC, Chen TX, et al: Discovery of tissuespecific exons using comprehensive human exon microarrays. Genome Biol 8: R64, 2007.

22. Kohno K, Chiba M, Murata S, et al: Identification of natural antisense transcripts involved in human colorectal cancer development. Int J Oncol 37: 1425-1432, 2010.

23. Skawran B, Steinemann D, Weigmann A, et al: Gene expression profiling in hepatocellular carcinoma: upregulation of genes in amplified chromosome regions. Mod Pathol 21: 505-516, 2008.

24. Tamori A, Yamanishi Y, Kawashima S, et al: Alteration of gene expression in human hepatocellular carcinoma with integrated hepatitis B virus DNA. Clin Cancer Res 11: 5821-5826, 2005.

25. Zhou Y, Zhou N, Fang W and Huo J: Overexpressed HDGF as an independent prognostic factor is involved in poor prognosis in Chinese patients with liver cancer. Diagn Pathol 5: 58, 2010.

26. Yoshioka S, Takemasa I, Nagano H, et al: Molecular prediction of early recurrence after resection of hepatocellular carcinoma. Eur J Cancer 45: 881-889, 2009.

27. Liu Y,Zhu X, Zhu J, et al: Identification of differential expression of genes in hepatocellular carcinoma by suppression subtractive hybridization combined cDNA microarray. Oncol Rep 18: 943-951, 2007.

28. Smith MW, Yue ZN, Geiss GK, et al: Identification of novel tumor markers in hepatitis $C$ virus-associated hepatocellular carcinoma. Cancer Res 63: 859-864, 2003. 
29. Inagaki Y, Yasui K, Endo M, et al: CREB3L4, INTS3, and SNAPAP are targets for the 1q21 amplicon frequently detected in hepatocellular carcinoma. Cancer Genet Cytogenet 180: 30-36, 2008.

30. Aoki Y, Cioca DP, Oidaira H, Kamiya J and Kiyosawa K: RNA interference may be more potent than antisense RNA in human cancer cell lines. Clin Exp Pharmacol Physiol 30: 96-102, 2003.

31. Wong CM, Lee JM, Ching YP, Jin DY and Ng IO: Genetic and epigenetic alterations of DLC-1 gene in hepatocellular carcinoma. Cancer Res 63: 7646-7651, 2003.

32. Matsukura S, Soejima $\mathrm{H}$, Nakagawachi $\mathrm{T}$, et al: $\mathrm{CpG}$ methylation of MGMT and hMLH1 promoter in hepatocellular carcinoma associated with hepatitis viral infection. Br J Cancer 88: 521-529, 2003.

33. Matsumura T, Makino R and Mitamura K: Frequent downregulation of E-cadherin by genetic and epigenetic changes in the malignant progression of hepatocellular carcinomas. Clin Cancer Res 7: 594-599, 2001.

34. Lau WY, Lai PB, Leung MF, et al: Differential gene expression of hepatocellular carcinoma using cDNA microarray analysis. Oncol Res 12: 59-69, 2000.

35. Shirota Y, Kaneko S, Honda M, Kawai HF and Kobayashi K: Identification of differentially expressed genes in hepatocellular carcinoma with cDNA microarrays. Hepatology 33: 832-840, 2001.

36. Li Y, Tang R, Xu H, et al: Discovery and analysis of hepatocellular carcinoma genes using cDNA microarrays. J Cancer Res Clin Oncol 128: 369-379, 2002.

37. Chung EJ, Sung YK, Farooq M, et al: Gene expression profile analysis in human hepatocellular carcinoma by cDNA microarray. Mol Cells 14: 382-387, 2002.

38. Grigoriadis A, Oliver GR, Tanney A, et al: Identification of differentially expressed sense and antisense transcript pairs in breast epithelial tissues. BMC Genomics 10: 324, 2009.

39. Li X, Feng P, Ou J, et al: Dermatopontin is expressed in human liver and is downregulated in hepatocellular carcinoma. Biochemistry (Mosc) 74: 979-985, 2009.
40. Sun H, Chua MS, Yang D, Tsalenko A, Peter BJ and So S: Antibody arrays identify potential diagnostic markers of hepatocellular carcinoma. Biomark Insights 3: 1-18, 2008.

41. Tan MG, Kumarasinghe MP, Wang SM, Ooi LL, Aw SE and Hui KM: Modulation of iron-regulatory genes in human hepatocellular carcinoma and its physiological consequences. Exp Biol Med (Maywood) 234: 693-702, 2009.

42. Kobayashi Y, Higashi T, Nouso K, et al: Expression of MAGE, GAGE and BAGE genes in human liver diseases: utility as molecular markers for hepatocellular carcinoma. J Hepatol 32: 612-617, 2000.

43. Mahmoudi S, Henriksson S, Corcoran M, Mendez-Vidal C, Wiman KG and Farnebo M: Wrap53, a natural p53 antisense transcript required for p53 induction upon DNA damage. Mol Cell 33: 462-471, 2009.

44. Faghihi MA, Zhang M, Huang J, et al: Evidence for natural antisense transcript-mediated inhibition of microRNA function. Genome Biol 11: R56, 2010.

45. Vogelstein B, Fearon ER, Hamilton SR, et al: Genetic alterations during colorectal-tumor development. N Engl J Med 319: 525-532, 1988.

46. Bruix J and Llovet JM: Prognostic prediction and treatment strategy in hepatocellular carcinoma. Hepatology 35: 519-524, 2002.

47. Kojiro M: Pathology of early hepatocellular carcinoma: progression from early to advanced. Hepatogastroenterology 45 (Suppl 3): 1203-1205, 1998

48. Midorikawa Y, Tsutsumi S, Taniguchi $\mathrm{H}$, et al: Identification of genes associated with dedifferentiation of hepatocellular carcinoma with expression profiling analysis. Jpn J Cancer Res 93: 636-643, 2002

49. Honda M, Sakai Y, Yamashita T, et al: Differential gene expression profiling in blood from patients with digestive system cancers. Biochem Biophys Res Commun 400: 7-15, 2010. 